\title{
Tschechische, österreichische und deutsche Kulturstandards in der Wirtschaftskooperation
}

\author{
Gerhard Fink, Ivan Nový \& Sylvia Schroll-Machl
}

\section{Einleitung}

In einer sich immer schneller internationalisierenden und globalisierenden Welt wächst die Anzahl der Personen, die beruflich und privat mit Menschen aus anderen Kulturen zusammenarbeiten und zusammenleben wollen oder müssen. Die interkulturellen Probleme, die dadurch auf die Betroffenen zukommen, werden zunehmend wissenschaftlich erforscht, um sie durch gegenseitiges Verständnis überwindbar zu machen. Der folgende Artikel hat nicht spektakulär verschiedene Kulturen zum Inhalt, sondern drei Nachbarn.

Der Ansatz, der zur Erforschung der zwischen Tschechen, Österreichern und Deutschen bestehenden Kulturunterschiede gewählt wurde, ist dabei dem Kulturstandardansatz nach Thomas (1996) verpflichtet sowie der multiperspektivischen Theorie nach Demorgon (1996). In einer neuartigen Kombination beider Herangehensweisen werden Kulturstandards in der Logik „adaptiver Gegensätze“ als Ausprägungen von Orientierungssystemen auf polaren Dimensionen verstanden. (Schroll-Machl \& Novy, 2000). D. h. in einem binationalen Kulturvergleich werden sie als mehr oder weniger gegensätzliche Kontrastpaare definiert, in einem trinationalen Vergleich als Abstufungen auf einer bipolaren Skala, wobei sie hier jeweils Ausprägungsformen eher in der Nähe des einen oder des gegenteiligen Pols oder dazwischen beschreiben. Die Pole werden dabei als in einem dynamischen Verhältnis zueinander stehend aufgefasst, weil jede Kultur prinzipiell beide Pole realisiert, allerdings in einem unterschiedlichen Mischungsverhältnis. Somit sind die Gewichtungen zwischen den untersuchten Kulturen verschieden: Die einen verhalten sich mehr „so als so“, die anderen mehr „so als anders“" und ein Kulturstandard beschreibt nun dieses spezifische Mischungsverhältnis. Denn konkrete Individuen „oszillieren“ in ihrem Verhalten zwischen den Polen in Abhängigkeit von Situationen und Zielen. Deswegen stellen Kulturstandards auf einer kollektiven Ebene die Mediane der individuellen Oszillationsprozesse der Angehörigen einer Kultur dar.

Die Ergebnisse beruhen auf einer qualitativen Studie zur Erhebung tschechischer, deutscher und österreichischer Kulturstandards. Die Studie folgte einem dreigliedrigen Design: (1) In 50 narrativen Interviews wurden zwischen 
1996 und 1997 in Deutschland, Österreich und der tschechischen Republik Kritische Ereignisse in der geschäftlichen Interaktion zwischen Tschechen, Deutschen und Österreichern erhoben. (2) Mit diesen kritischen Ereignissen wurden dann in einem mehrstufigen Verfahren mit einer Methodentriangulation (schriftliche Befragung, Gruppendiskussion, Experteninterviews) die jeweils eigenkulturellen Attributionen zu den Beobachtungen der ,anderen“ erfaßt. (3) Das so gewonnene Datenmaterial wurde schließlich einer qualitativen Inhaltsanalyse unterzogen, deren Ergebnis die als Kulturstandards bezeichneten Kategorien waren. - Die folgenden Ausführungen beschränken sich auf eine knappe Darstellung der Resultate dieser Studie: tschechische, österreichische und deutsche Kulturstandards im Vergleich. Eine ausführlichere Publikation findet sich in Novy'\& Schroll-Machl (1999) bzw. Schroll-Machl \& Nový (2000).

\section{Tschechische, deutsche und österreichische kulturelle Orientierung}

\section{Adaptiver Gegensatz: Personbezug - Sachbezug}

\section{Tendenz der Tschechen: Personbezug}

Der Kulturstandard „tendenzieller Personbezug“ beschreibt die Tatsache, dass Tschechen in der Interaktion und Kommunikation dem Beziehungsaspekt den Vorrang vor dem Sachaspekt einräumen. Das heißt, dass Tschechen stets die jeweils Agierenden stärker und bedeutsamer wahrnehmen als den Inhalt ihres Tuns. Die Sachebene rangiert daher in jeder Interaktion an der zweiten Stelle. Man sucht nach persönlichen Ansatzpunkten, bemüht sich, nett zu sein, ist aber auch selbst leicht in seinem Wohlbefinden kränkbar. Aus dem Grund bemühen sich Tschechen beispielsweise bei jeder Interaktion, eine menschlich möglichst angenehme Atmosphäre herzustellen - das tut der eigenen wie der anderen Person gut. Einmal geschaffene gute Beziehungen will man sich dann erhalten und man pflegt sie.

\section{Tendenz der (West)deutschen: Sachbezug}

Für die berufliche Zusammenarbeit ist unter Deutschen dagegen die Sache, um die es geht, die Rollen und die Fachkompetenz der Beteiligten ausschlaggebend. Die Motivation zum gemeinsamen Tun entspringt der Sachlage, evtl. den Sachzwängen. In geschäftlichen Besprechungen „kommt man zur Sache“ und „bleibt bei der Sache“. Ein „sachliches“ Verhalten ist es, was Deutsche als professionell schätzen: Man zeigt sich zielorientiert und argumentiert mit Fakten. Wenn man sich kennt oder gar mag, ist das ein angenehmer Nebeneffekt, doch das ist nicht primär relevant. Die Sache ist der Dreh- und 
Angelpunkt des Tuns und bestimmt auch den Kommunikationsstil. Etwaige Empfindlichkeiten sind da schon mal hintan zustellen.

\section{Tendenz der Österreicher: Weniger Sachbezug, mehr Personbezug}

Der Sachbezug ist bei Österreichern deutlich weniger betont als bei Deutschen, der Personbezug deutlich mehr. Sie liegen quasi „Zwischen“ Tschechen und Deutschen. Für Österreicher ist die Fachkompetenz wichtig, aber nicht alleine ausschlaggebend. Ihr Kommunikationsstil berücksichtigt stets auch die Beziehungsebene: Sie bemühen sich um Höflichkeit und das Hinterlassen eines angenehmen, charmanten Eindrucks, um Freundlichkeit, Hilfsbereitschaft, Gastfreundschaft und Rücksichtnahme. Sie argumentieren weniger "hart" als Deutsche. In der Zusammenarbeit in einem Team ist für sie das soziale Element des Sich-Verstehens eine wesentliche Voraussetzung für Kooperation.

\section{Adaptiver Gegensatz: Abwertung von Strukturen/Improvisationsliebe versus Aufwertung von Strukturen/Unsicherheitsvermeidung}

\section{Tendenz der Tschechen: Abwertung von Strukturen / Improvisationsliebe}

Tschechen stehen Strukturen skeptisch gegenüber. Statt dessen lieben sie es, zu improvisieren. Sie halten es für eine ihrer charakteristischen Eigenschaften, flexibel, geschmeidig, findig zu sein. Diese Eigenschaft erfüllt sie mit Stolz: kreativ sein, gestalten, spielen - das bevorzugen sie allemal. Das ist - so sind sie weithin überzeugt - auch der Boden, auf dem neue und gute Ideen gedeihen können.

Während für Deutsche jede Form von Plan hilfreich ist, weil er Zeit und Inhalte (Sache) organisiert, erleben Tschechen einen Plan als Einschränkung: Er organisiert in ihren Augen nicht die Sache, sondern die Person(!). Deshalb ist es verständlich, dass sie ihn tendenziell ablehnen. Tschechen gehen zudem davon aus, dass sich irgend jemand am grünen Tisch diesen Plan oder diese Norm willkürlich ausgedacht hat, ohne eine Ahnung davon zu haben, ob das tatsächlich sinnvoll oder gar notwendig ist. Normen, Vorschriften und Gesetze werden a priori sehr oft für dumm und unsinnig gehalten. Wer sich nun daran hält, erweist sich als einfältig und nicht-mitdenkend. Intelligenz besteht darin, sie zu umgehen. Ob das in der jeweiligen konkreten Situation stimmt, sei dahingestellt. Tatsache ist, innerlich erfolgt eine Abwertung der außen wahrgenommenen Struktur.

Das Besondere an diesem Kulturstandard ist neben der Fähigkeit, auf improvisierende Art handeln zu können, das innerliche, subjektive Erleben der Improvisation als Freiheit und Souveränität. Weil das so ist, hat dieser Kulturstandard eine enorme Reichweite: Tschechen zeigen geradezu ein prinzipielles Misstrauen und grundsätzliche Zweifel an allem (Gegenständen, Verfahren, Personen...), was sie nicht kennen und ihnen neu ist. Sie werden das 
Neue untersuchen und überprüfen, vielleicht etwas abändern und dann entscheiden, ob sie dieses Neue für gut halten oder nicht. Man will sich zum einen nicht übervorteilen lassen, sagen sie. Sie seien nun mal in der schwächeren Position und müssten sehr vorsichtig sein. Also müsse man etwas erst ausprobieren, das Resultat abwarten und dann sein Handeln aufgrund der Ergebnisse fortsetzen oder nicht. Man will sich zum anderen, wo immer man Einschränkung wittert, improvisierend verhalten zur Rettung der inneren Würde als freier Mensch. Man will damit sowohl das Gefühl der Wahlfreiheit seiner Handlungen haben wie auch sich selbst und anderen seine Würde und Intelligenz beweisen.

Dass diese Eigenschaften auf Kosten der Qualität, der Perfektion, der Optimalität der Sache gehen können, ist Tschechen kein Problem. Hier zeigen sie sich großzügig.

Die „Abwertung von Strukturen“ beinhaltet daher folgende Qualitäten:

1. Findigkeit zur Wiederherstellung der als bedroht erlebten Freiheit (sog. Reaktanzphänomene)

2. Kreativität / Einfallsreichtum / Improvisation i.e.S.

3. geringerer Qualitätsanspruch

4. Gelassenheit.

\section{Tendenz der (West)Deutschen: Aufwertung von Strukturen / Unsicherheitsvermeidung}

Deutsche lieben Strukturen. Dahinter steckt:

Deutsche streben in ihrem beruflichen Handeln nach einem „Optimum“, das sie mit Hilfe von Strukturen erreichen zu können glauben. Sie wollen die Dinge, die sie tun, möglichst gut machen und sich einem Optimum/Maximum möglichst weitgehend annähern. Sie stecken sich daher qualitativ hochwertige Ziele (hohe Produktqualität; hoher Organisationsgrad der Logistik usw.) und wollen dann einen möglichst reibungslosen, gangbaren und effektiven Weg zu diesem Ziel beschreiten. Als die Art und Weise, wie dieses Optimum zu erreichen ist, gelten Strukturen, Systeme und Normen, die Uneindeutigkeiten, Unsicherheiten und damit Störungen ausschalten sollen.

Im Kontrast zur tschechischen Skepsis liegt die entscheidende Grundeinstellung Deutscher bzgl. beruflicher Normen und Systeme nun des weiteren darin, dass man Strukturen i.a. als ,geronnene Erfahrung“ betrachtet. Hier hat sich nicht jemand willkürlich eine Norm ausgedacht, die im Grunde sinnlos ist und auch ganz anders sein könnte, sondern hier schlugen sich die Erfahrungen vieler nieder, die bereits an diesem und ganz ähnlich gelagerten Problemen gearbeitet haben. Der nunmehr als Struktur vorhandene Weg erwies sich dabei als gut und effektiv und deshalb ist man gewillt, diesen Weg auch künftig zu beschreiten. 
Das gilt für sämtliche Tätigkeiten in der Produktion, aber auch für viele Verwaltungsabläufe oder sonstige, irgendwie auf Routine basierenden Arbeiten. Hat jemand Kritik an diesen Verfahren zu äußern, kann er das als Verbesserungsvorschlag und als Weiterentwicklung tun. Dass ein einzelner jedoch klüger wäre als die „geronnene Erfahrung“ vieler, die ebenfalls Fachleute auf ihrem Gebiet sind bzw. waren, wird nur in Ausnahmefällen und aufgrund sehr stichhaltiger Argumente akzeptiert.

Mit anderen Worten: Hinter ihren Systemen und Normen sehen Deutsche i. a. viel Sinn. Die Normen haben geradezu Symbolcharakter für „beständige deutsche Wertarbeit" oder für Fortschritt im Sinne einer kontinuierlichen, verbessernden Veränderung.

Der Kulturstandard „Aufwertung von Strukturen“ ist somit folgendermaßen zu definieren:

Um (1) das Erreichen ihres relativ hohen Qualitätsanspruchs absichern zu können, sind Deutsche (2) planerisch, strukturierend und organisierend tätig (3) bis ins Detail. Das Grundmotiv ist dabei, ein Maximum an Orientierung und Unsicherheitsvermeidung sowie eine Risikominimierung zu erreichen. Das ist im beruflichen Feld gleichbedeutend mit Qualität: nichts Wichtiges übersehen, keinen Fehler machen.

\section{Tendenz der Österreicher: Gelassene Wertschätzung von Strukturen}

Prinzipiell ähneln sich auf dieser Dimension Österreicher und Deutsche. Die Organisationsliebe bis ins Detail ist allerdings etwas weniger ausgeprägt, ebenso das rigide Festhalten an einer Struktur: Pläne werden flexibler gehandhabt, Ziele gelassener verfolgt. - Die interviewten Tschechen berichteten über Deutsche und Österreicher die gleichen kritischen Ereignisse, der Unterschied ergab sich nur in einigen Teilbereichen im Vergleich der eigenkulturellen Attributionen der Österreicher mit denen der Deutschen, wenn sie diese Ereignisse aus ihrer eigenkulturellen Sicht erklärten.

\section{Adaptiver Gegensatz: Konsekutivität versus Simultanität}

\section{Tendenz der Tschechen: Simultanität}

Simultanität bedeutet, dass Tschechen mehrere Dinge zur gleichen Zeit tun und sich keinesfalls nur auf eines beschränken. Tschechen lieben es, vieles parallel zu machen und zwischen den Handlungssträngen je nach (subjektiver) aktueller Priorität im Vertrauen auf ihre Improvisationsfähigkeit zu wechseln. Sie wollen keine Chance verpassen und halten Augen und Ohren offen. Ihre Zielstrebigkeit ist dabei, wenn kein Druck vorhanden ist, nicht sehr ausgeprägt. Sie bevorzugen statt dessen eine breitere Berücksichtigung mehrerer Schienen und Ideen ohne unbedingt eine rote Linie zu haben oder sich einem Ergebnis- und Terminzwang zu unterwerfen. 


\section{Tendenz der (West)Deutschen: Konsekutivität}

Zeit ist für Deutsche ein wichtiges Thema. Deutsche scheinen Tschechen von Terminen und Zeitplänen getrieben und auf Termineinhaltung geradezu versessen. Wie kommt das zustande?

Deutsche haben die Vorstellung, dass es am optimalsten wäre, das Leben auf eine Art organisieren zu können, in der man sich (1) über eine anstehende Handlung Gedanken machen und sie planen kann, (2) diese Planung dann ohne Unterbrechungen und Störungen abarbeiten kann, um (3) schließlich sein Ziel zu erreichen. Weil das aber nicht geht, sondern alle Menschen gezwungen sind, viele Dinge parallel zu machen, bemühen sich Deutsche, ihrem Ideal doch zumindest nahe zu kommen: Sie packen die Dinge in klare Zeitfenster und Zeiteinheiten, ordnen sie dann nacheinander an und erledigen sie - soweit wie möglich - in dieser Reihenfolge.

Dieser Umgang mit der Zeit hat folgende Konsequenzen:

1. Deutsche machen sich für jedes ihrer Vorhaben (z. B.: für das Projekt 1, das Projekt 2 usw.) einen (groben) Zeitplan, an den sie sich nun auch weitgehend halten.

2. Zur Koordination der diversen Zeitpläne für die parallelen Vorhaben (Projekt 1, Projekt 2) macht sich jede Person feinere Ablaufpläne, wann sie am besten was erledigt und setzt die Zeitfenster, die für die einzelnen Vorhaben vorgesehen sind, in eine sinnvolle, konsekutive Reihenfolge. (Zeitfenster 1 für Schritt 1 des Projekts 1; dann Zeitfenster 2 für Schritt 3 des Projekts 5; dann Zeitfenster 3 für Schritt 1 des Projekts 2; usw.)

3. Weil nun alle so denken und handeln, ist es bei gemeinsamen Vorhaben essentiell, dass sich die Individuen zeitlich koordinieren. Sie vereinbaren Termine. Diese Termine sind der Kitt für gemeinsame Aktivitäten, weil sie die individuellen Ablaufpläne und Zeitpläne verzahnen.

4. Termine sind verbindlich, denn sonst gerät das System aus den Fugen.

Zeitmanagement gilt damit als Voraussetzung für effektives Handeln überhaupt, aber ganz sicher als wesentlicher Bestandteil von Professionalität. Man muss in der Lage sein, sich zeitliche Strukturen zu geben, realistische Einschätzungen für die einzelnen Zeitfenster vorzunehmen und sich dann eiserner zeitlicher Disziplin zu unterwerfen. Und Zeit erhält einen enormen Symbolwert, denn sie zeigt die Wichtigkeit einer Sache und einer Person an. Zeitliche Zuverlässigkeit ist für den Aufbau von Vertrauen eine kaum zu überschätzende Variable.

\section{Tendenz der Österreicher: Konsekutivität}

Auf dieser adaptiven Achse unterscheiden sich Österreicher und Deutsche nur in der Weise, dass Österreicher auch zeitlichen Plänen mit etwas mehr 
Gelassenheit, d.h. etwas mehr Toleranz für Abweichungen, begegnen. Prinzipiell gilt ansonsten das, was zu den Deutschen ausgeführt wurde.

\section{Adaptiver Gegensatz: Personorientierte Kontrolle versus regelorientierte Kontrolle}

\section{Tendenz der Tschechen: Personorientierte Kontrolle}

Die Begriffe „regelorientierte Kontrolle“ und „personorientierte Kontrolle“ beschreiben, dass Deutsche eher dazu neigen, allgemein gültige Regeln und Gesetze $\mathrm{zu}$ befolgen, während Tschechen dazu tendieren, zugunsten persönlicher Interessen oder Beziehungen auch gegen Regeln zu verstoßen. Während das deutsche Verhalten sehr stark an Regeln orientiert ist und davon ausgeht, dass Normen und Vereinbarungen eingehalten werden müssen, legen Tschechen mehr Wert auf menschliche Beziehungen und auf subjektives Wohlbefinden. Bei ihnen verpflichtet Freundschaft und hat daher Priorität vor abstrakten Regeln. Die Frage heißt daher: Wann herrscht bei Tschechen internale Kontrolle und wann externale Kontrolle vor?

1. Internale Kontrolle, Selbststeuerung hinsichtlich bestehender, äußerer, abstrakter Strukturen findet man ...

a. ... bei Personen und in Situationen dann, wenn die Sache dem handelnden Individuum ein persönliches Anliegen zur Verfolgung eigener Interessen ist;

b. ... wenn sich ein Individuum einer für sie relevanten Person auf einer solch guten und tragfähigen Beziehungsebene verbunden fühlt, dass es diese Person nicht enttäuschen möchte;

c. ... wenn eine hohe Identifikation mit der Sache herrscht.

In allen Fällen sind also persönliche Motive ausschlaggebend, nicht die Sache! Die Personorientierung gibt den Ton an!

2. Externale Kontrolle dominiert die Sachebene und die sie repräsentierende Struktur in den sonstigen Fällen: Ein „Pflichtbewusstsein“ gegenüber objektiven Regeln, Vereinbarungen, Normen usw. ist wenig ausgeprägt. Wenn bzw. solange keine oder nur eine zu geringe persönliche Motivation herzustellen ist, muss die Sachebene external kontrolliert werden! Schließlich gilt: Man respektiert Regeln nur, wenn es unumgänglich oder vorteilhaft ist.

\section{Tendenz der (West)Deutschen: regelorientierte Kontrolle}

Deutsche haben eine starke Identifikation mit der eigenen beruflichen Tätigkeit. Sie nehmen ihre Arbeit, ihre Rolle, ihre Aufgabe und ihre damit verbundene Verantwortung sehr ernst. Sie möchten das, was sie machen, gut machen und sind konzentriert bei der Sache. 
Zunächst einmal planen, organisieren, strukturieren sie. Das machen sie nicht zum Vergnügen, sondern aus der Überzeugung heraus, dass so die Aufgaben am besten bewältigt werden können (vgl. Aufwertung von Strukturen). Dass diese Strukturen nun in die Tat umgesetzt werden, hat eine zentrale Voraussetzung, die der Inhalt dieses Kulturstandards ist: Alle Beteiligten haben verlässlich zu sein. Eine Sache ist organisiert und jetzt wird von allen erwartet, dass sie sich korrekt an ihre Zuständigkeit halten und ihre Aufgabe erfüllen. Nur in diesem Zusammenspiel aller funktioniert das System. Das bedeutet, dass alle den im jeweiligen Kontext vorhandenen Normen, Systemen, Strukturen Folge leisten.

Es ist somit notwendig:

- sich im beruflichen Feld an Kompetenzen und Rollen zu halten;

- Absprachen, Vereinbarungen, Zusagen und Versprechen einzuhalten;

- Entscheidungen durchzuführen;

- Vorgaben exakt einzuhalten;

- zeitliche Zuverlässigkeit und Pünktlichkeit zu zeigen;

- den eigenen Handlungsspielraum als Verantwortungsspielraum wahrzunehmen und aktiv die nötige Initiative ergreifen.

Geschieht das, gilt jemand als zuverlässig, korrekt, gewissenhaft und er ist ein geschätzter Mitarbeiter oder Kollege, ein Vollprofi, wie man ihn sich wünscht und er verdient Vertrauen.

Diese Verlässlichkeit wird nun nicht vorrangig dadurch erreicht, dass es Instanzen gibt, die von außen kontrollieren, sondern dass jeder an seinem Platz aus sich heraus das tut, was von ihm erwartet wird. Tschechen formulieren das so: „Deutsche machen vieles ohne ersichtlichen Zwang dazu.“ Der Handelnde hat nämlich gar nicht mehr das Gefühl, dass sein Handeln andere von ihm erwarten, sondern es ist ihm selbstverständlich, das zu tun. Er hat sich im Prozess der Planung, der Strukturierung oder als er die Stelle antrat, damit bereits identifiziert. Im Fachbegriff spricht man von ,internalisierter Kontrolle“: Per Einsicht in die „Notwendigkeit“ bzw. Optimalität bestimmter Regelungen oder Verfahrensweisen kontrolliert sich ein Individuum weitgehend selbst. Es hält sich dabei entweder an vorgegebene Normen oder an selbst erstellte Pläne. Bei Verstößen oder Störungen kommt es daher nicht nur zu Konflikten mit einer Kontrollinstanz, z.B. dem Chef, sondern zu internen Konflikten und zu Gewissenskonflikten, weil man mit sich selbst unzufrieden ist. Man wurde gewissermaßen zum „Überzeugungstäter“.

Weil hier Strukturen, Normen, „Objektives“ internalisiert werden, besteht die deutsche Zuverlässigkeit gegenüber der Sache (vgl. Sachorientierung)! Die Beziehungen, die zu den beteiligten Personen existieren, beeinträchtigen oder fördern die gezeigte Gewissenhaftigkeit nicht. Ob der Chef sympathisch ist oder 
nicht, ob man sich mit seinen Kollegen wohlfühle oder nicht - man hat die Aufgabe zu erledigen. Und man will das auch, denn man findet die Sache im Prinzip gut, sonst wäre man nicht an dieser Stelle und nicht in diesem Job. Das Pflichtbewusstsein gilt somit in erster Linie den konkreten Vorgaben, die Loyalität der Firma, bei der man (gerade) arbeitet.

Auch das eigene, subjektive Wohlbefinden ist hintan zu stellen: Ob jemand Lust hat oder nicht, ob er gerade von Problemen heimgesucht ist, die ihm viel Energie abverlangen, ob es ihm sehr viel Mühe abverlangt oder Spaß macht, spielt keine Rolle: Er hat die Selbstdisziplin aufzubringen, sein Bestes zu geben. Denn er hat Ja gesagt zu dieser Vereinbarung oder dieser Stelle und nun steht er in Pflicht und Verantwortung. Selbstdisziplin und Härte zu sich selbst sind die Innenseite der Gewissenhaftigkeit.

Deutsche lieben keine Ausnahmen. $\mathrm{Zu}$ der bislang beschrieben Funktionalität von Strukturierung und Internalisierung gesellt sich eine weitere - soziale Bedeutung: Deutsche assoziieren mit ,gleichen Normen“ für alle auch „Gerechtigkeit“, d. h. gleiche Behandlung für alle hinsichtlich der Chancen und Rechte, aber auch der Sanktionen. Ausnahmen, Sondervereinbarungen, Abweichungen „bevorzugen“ aus deutscher Sicht den, dem sie zugestanden werden. Und das halten sie ,im Prinzip“ für unfair. Wenn Ausnahmen gemacht werden, dann bedarf es dazu einer zwingend einsichtigen Begründung oder der zuverlässigen Einschätzung der betreffenden Person als sehr verantwortungsbewusst, was garantiert, dass sie sich sonst selbstverständlich an die Normen hält.

\section{Tendenz der Österreicher: Abgeschwächte regelorientierte Kontrolle}

Im Vergleich zu Tschechen gilt für Österreicher im Grunde dasselbe wie für Deutsche. Im Vergleich $\mathrm{zu}$ den Deutschen ist jedoch der Grad der Internalisierung abgeschwächter: Österreicher bezweifeln die Vernünftigkeit von Regeln eher. Zudem bewahren sie sich stets eine gewisse innere Distanz zu den herrschenden Regeln und sind daher leichter bereit, in begründeten Fällen sich und anderen „Ausnahmen“ einzuräumen. Sie brechen eher einmal eine Regel und erscheinen Tschechen damit weniger "streng“ als Deutsche. Wiederum ergaben sich auf dieser Dimension nur graduelle Abweichungen der österreichischen eigenkulturellen Attributionen von den deutschen eigenkulturellen Attributionen. 


\section{Adaptiver Gegensatz: Diffusion von Lebens- und Persönlichkeitsbereichen versus Trennung von Lebens- und Persönlichkeitsbereichen}

\section{Tendenz der Tschechen: Diffusion von Lebens- und Persönlichkeitsbereichen}

Kulturen werden in „spezifische“ und „diffuse“ eingeteilt. Damit wird das Maß der Betroffenheit im Umgang mit anderen Menschen bezeichnet, d.h. es wird erfasst, ob man Menschen in bestimmten, „spezifischen“ Lebensbereichen und Aspekten ihrer Persönlichkeit begegnet oder ob man ihnen eher ganzheitlich, „diffus“ gegenübertritt. Im ersteren Fall sind die Lebens- und Persönlichkeitsbereiche analog einer biologischen Zellwand relativ undurchlässig und getrennt, im zweiten Fall hochgradig durchlässig. Die Tschechen zeigen deutliche Merkmale der Diffusion. Beobachtbar ist bei ihnen eine Vermischung der Persönlichkeitsbereiche „Emotionalität-Rationalität“ sowie der Lebensbereiche „Beruf - privat“, „Rolle-Person“ und „formelleinformelle Strukturen“ - und zwar in allen Stadien von Nähe und Bekanntschaft.

\section{Tendenz der (West)Deutschen: Trennung von Persönlichkeits- und Lebensbereichen}

Deutsche nehmen eine strikte Trennung der verschiedenen Bereiche ihres Lebens vor. Sie differenzieren ihr Verhalten sowohl deutlich danach, in welcher Sphäre sie mit einer anderen Person zu tun haben wie auch danach, wie nahe sie einer anderen Person stehen.

Die Unterscheidung folgender Sphären ist daher wesentlich:

- Beruf - privat

Deutsche arbeiten während der Arbeit und „leben“ in ihrer Freizeit, d.h. nach Feierabend, am Wochenende, im Urlaub. In der Arbeit hat die Arbeit Vorrang und alles andere tritt an die zweite Stelle. Im Privatleben nehmen Beziehungen, Familie, Freunde, persönliche Neigungen und Interessen die ganze Person in Anspruch. Im Beruf ist man sachorientiert, privat beziehungsorientiert. Im Beruf ist man zielstrebig, privat will und muss man entspannen. Im Beruf widmet man sich den jeweiligen Sachinhalten, im Privatleben fröhnt man unter Umständen ganz anderen Neigungen und schafft seinem Gemüt Ausgleich. Manchmal scheint es, als hätte man mit zwei verschiedenen Menschen zu tun - im äußeren Erscheinungsbild, im Verhalten, in der Stimmung.

- Emotionalität - Rationalität

Deutsche bemühen sich, ihre Gefühle und die „objektiven Fakten“ auseinanderzuhalten. Dabei ist das Vorherrschen der Rationalität vor allem im Berufsleben angesagt, wo es als professionell gilt, sich sachlich zu zeigen (vgl. Sachbezug) und Gefühle in mancherlei Hinsicht fast Schwäche bedeuten. Rationalität ist somit der Persönlichkeitsbereich, der beruflich aktiviert wird und 
die Basis für die Sachorientierung darstellt. Emotionalität ist dagegen im Privatleben dominanter. Jetzt ist wichtig, Mitgefühl mit und Verständnis für andere zu haben sowie sich seiner eigenen Gefühle bewusst zu sein und ihnen freieren Lauf zu lassen. Doch immer dann, wenn es um heikle Fragen geht, wird unterschieden zwischen dem, was man sich ,rational“ zu einer Sache denkt, und dem, was man „emotional“ „aus dem Bauch heraus“ meint. Beides ist dann gegeneinander abzuwägen, um zu handeln.

- Rolle - Person

Deutsche definieren die Rollen, die zu bestimmten Positionen gehören, klar. Professionalität bedeutet, man weiß um seine Rolle in allen Facetten - bis hin zu Kleinigkeiten. Und man hält diese Rolle auch ein. Beruflich heißt das: Man ist korrekt und in der Sache engagiert zugleich, angemessen distanziert und mit entsprechender fachlicher Qualifikation. Zeigt man darüber hinaus gehendes Verhalten, läuft man Gefahr ,aus der Rolle zu fallen“, was meist nicht positiv bewertet wird. Man ist weder zu enthusiastisch noch beleidigend. Die Person, die hinter der Rolle steht, ist häufig in vielerlei Hinsicht schillernder. Doch sie kann, will sie beruflich anerkannt sein, nur einen Teil ihrer Persönlichkeit in ihrer Rolle ausleben: am besten die Seiten, die der Rolle förderlich sind und den Rolleninhaber damit überzeugend oder gar charismatisch erscheinen lassen.

- formell - informell

Deutsche trennen auch zwischen formellen und informellen Settings. Die wünschenswerte Norm heißt dabei: Die wichtigen Dinge laufen in den formellen Kanälen. Damit sind sie einsehbar, nachvollziehbar und einfacher zu handhaben. Was Deutsche deshalb analog dem Kulturstandard „Aufwertung von Strukturen“ organisieren, das hat auch für den Alltag tatsächliche Bedeutung. Informelle Settings haben eher den Geruch des Verdeckten, Hinterhältigen, Illegalen, Halbseidenen, mitunter sogar Intriganten - eben einer Struktur, die eigentlich nicht sein sollte. Ihrer bedient man sich, wenn es um Kampf und Macht geht und die, die darin involviert sind, lassen das am besten nicht offenkundig werden.

- Distanzregulierung:

Außerdem spielt für die Art des Kontakts zu Deutschen Nähe eine entscheidende Rolle. Es sind bei ein- und derselben Person ganz unterschiedliche Verhaltensweisen beobachtbar, je nachdem, ob ihr Interaktionspartner ein Fremder, ein Bekannter / Kollege, ein guter Bekannter oder ein echter Freund ist. Die Entwicklung von Freundschaften ist dabei der (angenehme) Ausnahmefall. Als durchgängiges Muster kann für Deutsche gesagt werden, dass sich (a) der Kontakt vom Distanzierten und Formellen zum Vertrauten hin bewegt, dass (b) die anfängliche Sachlichkeit und Rationalität zunehmend größerer Emotionalität, Herzlichkeit und Personorientierung weicht, dass (c) Nähe eine „Herzenssache“ und nicht von Zweckrationalität bestimmt ist. Die Annäherung erfolgt Schritt für Schritt in den Stufen 
1. neutrales Verhalten zu Beginn

2. schrittweises Sichnäherkommen mit zunehmender emotionaler Öffnung

3. Freundlichkeit bis Herzlichkeit.

\section{Tendenz der Österreicher: Balance}

Österreicher zeigen gemäß ihres, im Vergleich zu Deutschen, weit höheren Personbezugs eine „Diffusion von Persönlichkeits- und Lebensbereichen“, jedoch gemäß ihres, im Vergleich zu Tschechen höheren Sachbezugs eine deutlichere „Trennung von Persönlichkeits- und Lebensbereichen“. Betroffen sind davon vor allem die Persönlichkeitsbereiche „Emotionalität - Rationalität“, sowie die Lebensbereiche „formell-informell“. Österreicher trennen Gefühle und „objektive Fakten“ weniger als Deutsche. Informelle Kontakte pflegen sie ähnlich intensiv wie Tschechen. Allerdings dient die Pflege dieser Beziehungen deutlicher den Personen und der Intensivierung des emotionalen Kontakts, als dass sie als Ersatz für die formelle Ebene fungiert. Beide Ebenen werden von Österreichern parallel bedient. Bezüglich der Distanzregulierung gilt für Österreicher ähnlich den Tschechen, wenn auch weniger ausgeprägt, ein größeres anfängliches Misstrauen und ausgeprägtere Vorsicht, nicht betrogen zu werden.

\section{Adaptiver Gegensatz: „Starker Kontext“ versus „schwacher Kontext“}

\section{Tendenz der Tschechen: Starker Kontext}

Der Fachbegriff „Kontext" meint, dass in Kulturen die Anteile des explizit und eindeutig Gesagten im Verhältnis zur Gesamtinformation, die in einer Situation enthalten ist, verschieden groß ist. Ist der Anteil der nicht-sprachlichen Botschaften hoch, dann handelt es sich um einen starken bzw. Hoch-Kontext. Ist der Anteil des verbal Formulierten und Nicht-Interpretationsbedürftigen hoch und damit der Kontextanteil gering, dann spricht man von einem schwachen oder Niedrig-Kontext.

Hochkontext-Kommunikation charakterisiert die verbalen Begegnungen unter Tschechen. Sie bedienen sich eines Kommunikationsstils, der (1) in den Formulierungen indirekter ist und (2) impliziter ist, d.h. mehr mit zusätzlichen, nicht-sprachlichen Signalen arbeitet, die dem Kontext zur Interpretation des Gesagten entnommen werden müssen. Tschechen sagen deshalb, sie drücken sich „schlauer“ aus als Deutsche, denn sie müssen nicht alles, was sie mitteilen wollen, sagen. Man kann es der Situation durch genauer Wahrnehmung entnehmen, indem man den gesamten Zusammenhang dessen, was gesagt wurde, berücksichtigt: Wer sagte was wann zu wem wie unter welchen Umständen? 


\section{Tendenz der (West)Deutschen: schwacher Kontext}

Während Tschechen nur relativ wenig direkt sagen, sondern vieles in kontextuelle Signale verpacken, bevorzugen Deutsche einen Stil großer Direktheit und Explizitheit: Sie formulieren das, was ihnen wichtig ist, mit Worten und benennen die Sachverhalte dabei ungeschminkt. Die charakteristischen Elemente dieses Stils sind:

1. Das WAS steht im Vordergrund, das WIE ist sekundär. - Der Fokus der Deutschen ist nämlich, wie wir schon dargelegt haben, vor allem auf die Sachebene gerichtet, d.h. ihnen kommt es auf den Inhalt des Gesagten an. (vgl. Kulturstandard Sachorientierung)

2. Daher reden Deutsche direkt und undiplomatisch, aber ehrlich und aufrichtig, ganz so, wie sie etwas eben sehen.

3. Sie denken nicht daran, auf etwaige Empfindlichkeiten der Anwesenden besonders Rücksicht nehmen zu müssen. Und so können Ihre Aussagen verletzend wirken, obwohl das nicht so gemeint und beabsichtigt war. Schließlich handeln sie gemäß der „Trennung von Lebensbereichen“.

4. Interpretationsspielraum zu lassen, ist zudem nicht ihre Sache. Sie wollen sich präzise, klar und unmissverständlich ausdrücken und daher formulieren sie die Dinge, die sie mitteilen wollen, aus. Sie meinen das, was sie sagen; und sie sagen das, was sie meinen. Ergänzende Informationen braucht man nicht dazu zu nehmen, zusätzlich wahrzunehmen oder aus dem Kontext des Gesagten zu entschlüsseln, um im Bilde zu sein, was ihre Botschaft war.

5. Umgekehrt wird in die Dekodierung nur miteinbezogen, was ausdrücklich gesagt wird. Deutsche denken nicht daran, dass das, was man ihnen sagt, nur ein Teil der Botschaft sein könnte, die um weitere Signale ergänzt werden müsste, damit sie verstanden werden kann. Sie hören explizit gesprochene Worte, halten das gewohnheitsmäßig für den Inhalt, den man transportieren wollte und haben keine Ahnung, dass noch anderes zur zuverlässigen Entschlüsselung und Interpretation des Gesagten hinzu genommen werden müsste.

\section{Tendenz der Österreicher: Mittlerer Kontext}

Österreicher bedienen sich eines Kommunikationsstils, der im Vergleich zu Deutschen vor allem als relativ indirekt und „diplomatisch“ zu bezeichnen ist, im Vergleich zu Tschechen jedoch vorwiegend als relativ explizit. Eine wesentliche Rolle spielt dabei der Humor, der viele Aussagen entschärft und einen spielerischeren Umgang mit Mitteilungen ermöglicht. Die größere Indirektheit erlaubt es außerdem, nicht alle Karten offen zu legen, sondern sich in mancher Hinsicht bedeckt zu halten. 


\section{Adaptiver Gegensatz: Konfliktvermeidung versus Konfliktkonfrontation}

\section{Tendenz der Tschechen: Konfliktvermeidung}

Ein sehr schwieriges Feld in der Interaktion zwischen Tschechen und Deutschen ist das völlig andere Umgehen mit Konflikten. Tschechen sagen von sich, dass sie nicht (hart) diskutieren können, dass sie Probleme nicht besprechen können, ja dass ihnen solche Gespräche derart unangenehm sind, dass sie ihnen, wo immer möglich, ausweichen. Man gibt daher der Konfliktvermeidung auf alle Fälle Vorrang vor der Konfliktaustragung. Während also Deutsche Kritik aussprechen, Probleme analysieren, Schwierigkeiten und Unangenehmes beim Namen nennen, würden Tschechen am liebsten bei alledem im Boden versinken. Es ist ihnen nahezu unerträglich.

Wie also gehen Tschechen untereinander mit Konflikten um?

1. Zunächst einmal weichen sie der Thematisierung von Konflikten solange aus, wie es nur irgendwie geht. Es wird einfach so getan, als gäbe es keinen Konflikt. Man will während der Kontakte den Konflikt vergessen, ein möglichst angenehmes Beisammensein herstellen und genießen und damit wieder eine positive gemeinsame Basis schaffen. Der Konflikt wird glattgebügelt, so dass er die Beziehungsebene nicht mehr stört.

2. Die Signale, mit denen man Konflikte einer höheren, nicht zu leugnenden Eskalationsstufe kommuniziert, sind vor allem Kontext-Signale. Das tut man lange, ausgiebig, geduldig. Ein explizites Gespräch findet eher nicht statt. Wenn, dann werden die Konflikte dabei tendenziell bagatellisiert und ein 'kleines' Problem kann schon mal ein riesiges sein. Wenn Explizitheit wirklich einmal unumgänglich ist, dann werden Konflikte auf schriftlichem Wege thematisiert - beispielsweise per Fax zum vereinbarten Termin -, aber so gut wie nie mündlich.

3. Wird der Druck zu stark, so dass nichts mehr geschluckt werden kann, dann besteht die Gefahr der Explosion: Die Explosion kann (a) leise erfolgen, indem die Person plötzlich geht und sich ohne Begründung völlig aus der Situation zurückzieht. (b) Der „Knall“ kann laut sein und ebenfalls das Ende einer Beziehung bedeuten. Es kann aber auch der Rauch wieder abziehen und keiner ist nachtragend. Das kommt auf die Personen, die Situation und die Stärke der Betroffenheit an.

Für Deutsche sind nun die Stufen (1), (2) und (3a) nicht unterscheidbar, weil sie ja, wie dargestellt, die Kontextsignale der Tschechen meist nicht enträtseln können. Sie erleben nur Funkstille und ärgern sich über die tschechische „Passivität“". 


\section{Tendenz der (West)Deutschen: Konfliktkonfrontation}

Wie soeben erwähnt, gehen Deutsche dagegen im Einklang mit ihrem direkten Kommunikationsstil die Dinge geradezu ,frontal“ an.

- Hinsichtlich ihrer Selbstbehauptung sind sie nicht von Konfliktscheu heimgesucht. Sie kämpfen argumentativ für ihre Position. Sie schrecken vor einem klaren Nein nicht zurück. Wenn sie etwas wollen, dann sagen sie das so klar, dass andere es als „fordern“ erleben.

- Auch an der Kritik stößt sich so manche nicht-deutsche Seele. Das Kritikverhalten Deutscher ist nämlich vorrangig an der Sache ausgerichtet (vgl. Sachbezug). Sie sind überzeugt, dass sie lediglich eine Verfehlung kritisieren, aber nicht die Person. Seinen Fehler zuzugeben, ist dabei geschätzt.

- Wenn es Probleme zu lösen gilt, sind Deutsche davon überzeugt, dass nur durch eine klare Problemanalyse und ein gnadenloses Ansprechen von Schwachstellen eine Optimierung möglich ist: Erst wenn die Probleme erkannt sind, kann man an eine Fehlerbehebung gehen.

\section{Tendenz der Österreicher: Konfliktvermeidung}

Auch Österreicher scheinen sehr bemüht, Konflikte zu vermeiden, statt sie auszutragen. Es gilt die Norm, Konflikte solange nicht anzusprechen, solange sie (noch) nicht offen sind. Es gilt als klug, sich zur Konfliktvorbeugung nicht festzulegen, sondern alles lange offen zu lassen. Es gilt als bequemer, Konflikte $\mathrm{zu}$ umgehen. Außerdem droht die Gefahr, dass in einem Konflikt auch peinlicherweise verdeckte, unehrenhafte Ziele aufgedeckt werden könnten („Hinterfotzigkeit“). Wenn sie Kritik äußern, dann sehr „diplomatisch“, d.h. durch verschiedene Floskeln abgeschwächt, abgepuffert, abgemildert, gedämpft (vgl. Indirektheit).- Trotzdem leben auch Österreicher, wie Deutsche, negative Gefühle aus: sie granteln, raunzen, beschweren sich, tun das aber häufiger als Deutsche hinter dem Rücken derer, auf die sich die negativen Gefühle beziehen.

\section{Adaptiver Gegensatz: schwankende Selbstsicherheit versus stabile Selbstsicherheit}

\section{Tendenz der Tschechen: Schwankende Selbstsicherheit}

Tschechen unterliegen in ihrer Selbstsicherheit mitunter größeren Schwankungen. Sie pendeln zwischen Bescheidenheit und Understatement einerseits und Selbstüberschätzung und Übertreibung andererseits. Manchmal erscheinen sie fast unterwürfig, um dann wieder zu glauben, sie seien um Längen besser und anderen klar überlegen. Das gilt sowohl interindividuell, d.h. manche Tschechen zeigen ein eher zu großes und andere ein eher zu geringes 
Selbstbewusstsein; das gilt aber auch intraindividüll, so dass ein- und dieselbe Person mal in die eine Richtung und mal in die andere Richtung tendiert.

\section{Tendenz der (West)Deutschen : Stabile Selbstsicherheit}

Tschechen charakterisieren Deutsche stets als betont selbstsicher. In einer weniger schmeichelhaften Formulierung nennen sie sie arrogant. Deutsche selbst bezeichneten dieses ihr berufliches Verhalten als geprägt von Leistungsbewusstsein, Professionalismus, Kompetenz, Glaubwürdigkeit sowie Streben nach Akzeptanz, Ansehen, Anerkennung und Karriere. Und das sind für sie im Berufsleben alles positiv besetzte Begriffe.

Der Ausgangspunkt ist dabei vielfach der, dass Deutsche gerne den Eindruck erwecken, von einer Sache etwas zu verstehen und in einem/ihrem Gebiet Experten zu sein (vgl. Sachbezug). Und nun kommen Typiken des typisch deutschen Kommunikationsstils hinzu: Die Meinung zu sagen, zu widersprechen, im Brustton der Überzeugung zu reden. Und das alles ohne Signale von Angst. Eine weitere Steigerung stellt die Gewohnheit dar, ein gewisses Marketing für sich selbst, die Firma und das jeweilige Vorhaben und die erreichten Erfolge zu machen.

\section{Tendenz der Österreicher: stabile Selbstsicherheit}

Auch auf dieser Dimension war kein Unterschied zwischen Österreichern und Deutschen festzustellen. Weder gab es Kritische Ereignisse, die einen Unterschied nahe legen würden, noch zeigten sich in den eigenkulturellen Attributionen Differenzen zwischen Deutschen und Österreichern.

\section{Ausblick}

Die Ergebnisse dieser Untersuchungen korrespondieren durchaus mit vielen Aussagen auf der Ebene von Stereotypen: Österreicher erscheinen Tschechen viel sympathischer und näher als Deutsche; Österreicher nehmen sich selbst als eine sympathische Variante des Deutschtums wahr; Deutsche wissen, dass sie bei Tschechen wie bei Österreichern wenig beliebt sind und glauben auch von sich selbst, etliche nicht-liebenswerte Charakteristika zu besitzen; Deutsche finden Tschechen oft sympathisch, wenngleich undurchsichtig und vermissen bereits an Österreichern die gewohnte Eindeutigkeit und Klarheit. Gemäß der These, dass Ähnlichkeit Sympathie fördert, ist das erklärlich, weil Österreicher auf vielen Dimensionen zwischen den Deutschen und den Tschechen zu verorten sind. Dabei spielen vermutlich die Pole Sachbezug - Personbezug eine herausragende Rolle, weil sie in engem Zusammenhang zu allen Kulturstandards stehen. 


\section{Literatur}

Demorgon, J. \& Molz, M. (1996). Bedingungen und Auswirkungen der Analyse von Kultur(en) und interkulturellen Interaktionen. In: Alexander Thomas (Hrsg.), Psychologie interkulturellen Handelns, Göttingen: Hogrefe. S. 43-86.

Nový, I. \& Schroll-Machl, S. (1999). Interkulturní komunikace v rízeni a podnikání. Praha: Management Press.

Schroll-Machl, S.\& Nový, I. (2000). Perfekt geplant oder genial improvisiert? Kulturunterschiede in der deutsch-tschechischen Zusammenarbeit. Mering: Hampp.

Thomas, A. (Hrsg.) (1996). Psychologie interkulturellen Handelns. Göttingen: Hogrefe. 